\title{
Channel estimation in OFDM systems operating under high mobility using Wiener filter combined basis expansion model
}

\author{
Ke Zhong ${ }^{*}$, Xia Lei, Binhong Dong and Shaoqian Li
}

\begin{abstract}
In this article, we first thoroughly analyze Wiener filter combined least squares based channel estimation (WF-LS) and then illustrate its limitation in high-speed mobile environments. Based on the analysis, we propose to combine WF with basis expansion model (BEM) based channel estimation to deal with channel estimation in various mobile environments, especially in high-speed cases. The expression for Wiener filter combined BEM based channel estimation (WF-BEM) is derived and the result explicitly considers the effect of intercarrier interference (ICI) that occurs in orthogonal frequency division multiplexing (OFDM) systems when operating under high mobility. The simulation results demonstrate that our proposed WF-BEM is better than WF-LS in time-varying channels, and the performance improvement is significant especially in fast time-varying channels.
\end{abstract}

\section{Introduction}

Due to its high data rate transmission capability and its robustness to multipath delay spread, orthogonal frequency division multiplexing (OFDM) has been adopted in most parts of modern wireless communication sytstems, such as wireless local area networks (WLAN) [1], digital audio and video broadcasting [2], and so as OFDM being standardized for the future wireless communication systems, such as wireless metropolitan area networks (WiMAX) [3] and 3GPP long term evolution (LTE) [4]. On the other hand, with high-speed railway construction worldwide, the moving speed of high-speed train has been reported to be able to operate as high as more than $400 \mathrm{~km} / \mathrm{h}$, or, even higher on-board highspeed vehicles such as aircraft. Consequently, mobility support is widely regarded as one of the key features in the above-mentioned OFDM systems. Meanwhile, for coherent detection in a wireless communication system, channel state information is indispensable. Therefore, to ensure effective communication of OFDM systems operating in high speed scenarios or alternatively very fast time-varying wireless channels, channel estimation

\footnotetext{
* Correspondence: kezhong85@gmail.com

* Correspondence: kezhong85@gmail.com
The National Key Laboratory of Science and Technology on

Communications, University of Electronic Science and Technology of China, Chengdu 611731 P. R. China
}

(c) 2012 Zhong et al; licensee Springer. This is an Open Access article distributed under the terms of the Creative Commons Attribution License (http://creativecommons.org/licenses/by/2.0), which permits unrestricted use, distribution, and reproduction in any medium, provided the original work is properly cited.

method applicable to such high-speed environments has remained largely an open issue.

Although blind channel estimation methods can save bandwidth by avoiding the trans-mission of any pilot or training symbols, they must exploit the statistic of the received signals, either explicitly or implicitly, which requires the wireless channel is time invariant during several OFDM symbols (e.g., cyclic prefix (CP) [5] or virtual carriers [6] based blind channel estimation method, etc.). Therefore, blind channel estimation methods are only capable of tracking slow channel variations. In fast fading channels, they will suffer severe performance degradation. For channel estimation in wireless communication systems operating under high mobility, pilot symbols (i.e., symbols with known pilot tones) must be inserted periodically into the transmission frame to track fast channel variations (also called pilotassisted transmission). Channel estimation in fading channels with very high mobilities usually consists of two steps. As shown in Figure 1, channel estimation at pilot symbols is the first step in completing channel estimation in communication systems. The next step, we need to perform interpolation between the pilot symbols to obtain the channel estimate of data symbols (i.e., symbols only with unknown data subcarriers), which are transmitted between these pilot symbols. On one hand, traditional training symbols or pilots aided channel

\section{Springer}




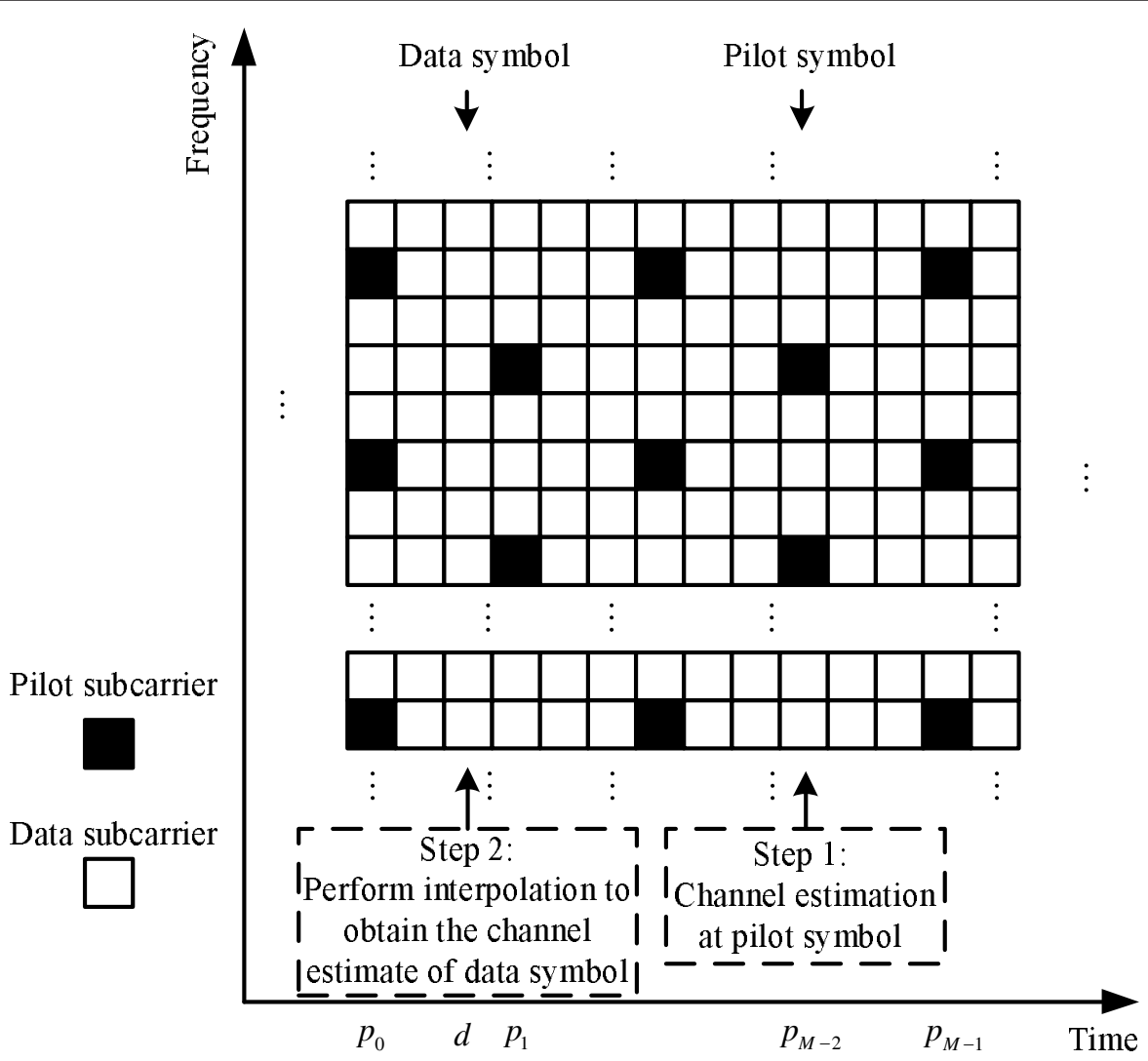

Figure 1 Frame structure and steps for channel estimation in OFDM systems.

estimation methods adopted to obtain the channel estimate of pilot symbols assume that the wireless channel is static during an OFDM symbol period, which means that the channel frequency response matrix is a diagonal matrix [7]. This assumption holds when the users are in indoor environments or is approximately valid in slow speed moving environments. Channel estimation problems in this situation have been well studied and solved in the literature. For example, least squares (LS) or minimum mean square error (MMSE) based channel estimation methods [8] can be used to estimate the diagonal of the channel frequency response matrix, or we can further proceed to eliminate noise in transformdomain [9]. On the other hand, Considering that Wiener filter (WF) is the best interpolation method at the time dimension in terms of minimizing MSE [10], Dong et al. [11] and Zheng and Xiao [12] derive the expression for Wiener filter combined LS based channel estimation (hereafter called WF-LS), combining LS based channel estimation with WF. The result is based on the assumption that the channel coefficients within an OFDM symbol period are constants and thus the effect of intercarrier interference (ICI) is completely neglected. Although the proposed WF-LS method can deal with slow to moderate fading channels, however, in time-varying channels, especially in fast time-varying cases, the latter assumption may be often violated in practice and the channel coefficients can vary in an OFDM symbol period, and thus ICI occurs, where the off-diagonal elements in the channel frequency response matrix represent ICI [7]. Therefore, the performance of WF-LS will degrade significantly or even lose its efficiency in this situation.

Basis expansion model (BEM) based channel estimation method can accurately estimate both slow and fast time-varying coefficients of the wireless channel in an OFDM symbol period using the coefficients of BEM that are far less than the length of wireless channel $[13,14]$. Combining BEM based channel estimation with WF, in this article we derive the expression for Wiener filter combined BEM based channel estimation (hereafter called WF-BEM), which explicitly considers the effect of ICI. The simulation results show that WF-BEM is better than WF-LS in time-varying channels, especially in fast time-varying cases.

The organization of this article is as follows. Section 2 describes the OFDM system model. Section 3 gives further analysis of WF-LS and then illustrates its 
limitation in time-varying channels. Section 4 derives the expression for WF-BEM based channel estimation. Section 5 gives some simulation results that demonstrate the effectiveness of the proposed algorithm. Finally, conclusions are drawn in the final Section 6.

Notation: Matrices and vectors are denoted by boldface letters. $\mathbf{A}(m, n)$ is the $(m, n)$-th entry of the matrix A. $\mathbf{x}(m)$ denotes the $m$-th element of the vector $\mathbf{x}$. A hat over a variable indicates an estimate of the variable (e.g., $\hat{H}), E\{\cdot\}$ denotes the expected-value operator. Superscripts $[\cdot]^{T},[\cdot]^{-1},[\cdot]^{H}$, and $[\cdot]^{*}$ denote the transpose, the matrix inversion, the Hermitian and the complex conjugate operations, respectively. $\mathbf{I}$ is the identity matrix.

\section{System description}

Let $\hat{\mathbf{P}}_{k}=\left[\hat{\mathbf{H}}_{p_{0}}(k) \hat{\mathbf{H}}_{p_{1}}(k) \ldots \hat{\mathbf{H}}_{p_{M-1}}(k)\right]^{T}$ be a column vector that represents the estimate of channel frequency response at the $k$-th subcarrier of pilot symbols in an OFDM system, $k=0,1, \ldots, N_{p}-1$ represents the pilot subcarrier index, which is evenly located in pilot symbols, $N_{p}$ is the number of pilot subcarriers in one pilot symbol, $p_{i}, i=0,1, \ldots, M-1$ represents the position of the $p_{i}$-th pilot symbol at the time dimension, $M$ represents the pilot symbol numbers (see Figure 1 for illustration), $\hat{\mathbf{H}}_{p_{i}}=\left[\hat{H}_{p_{i}}^{(0)} \hat{H}_{p_{i}}^{(1)} \ldots \hat{H}_{p_{i}}^{\left(N_{p}-1\right)}\right]^{T}$, and the pilot symbol insertion rate $r$ at the time dimension satisfies $r$ $\geq 2 f_{D} T, f_{D} T$ is the normalized Doppler frequency. Zadoff-Chu sequence [15] is adopted at the $p_{i}$-th pilot symbol $\mathbf{X}_{p_{i}}=\left[X_{p_{i}}^{(0)} X_{p_{i}}^{(1)} \ldots X_{p_{i}}^{\left(N_{p}-1\right)}\right]^{T}$ in this article, so $\left|\mathbf{X}_{p_{i}}(k)\right|^{2}=1$. The channel estimate $\hat{\mathbf{H}}_{d}(k)$ at the data symbol position $d$ can be estimated by interpolating $\hat{\mathbf{P}}_{k}$. Let the interpolation row vector be $\mathbf{C}_{d, k}$, and $\hat{\mathbf{H}}_{d}(k)$ can be obtained by $\hat{\mathbf{H}}_{d}(k)=\mathbf{C}_{d, k} \hat{\mathbf{P}}_{k}$. By minimizing the estimation MSE $\varepsilon_{d}=E\left\{\left|\mathbf{H}_{d}(k)-\hat{\mathbf{H}}_{d}(k)\right|^{2}\right\}$, namely,

$$
\underset{\mathbf{C}_{d, k}}{\arg \min } E\left\{\left|\mathbf{H}_{d}(k)-\hat{\mathbf{H}}_{d}(k)\right|^{2}\right\}
$$

the optimal interpolation vector, i.e., the WF can be obtained as [10]

$$
\hat{\mathbf{C}}_{d, k}^{W F}=E\left\{\mathbf{H}_{d}(k) \hat{\mathbf{P}}_{k}^{H}\right\} E\left\{\hat{\mathbf{P}}_{k} \hat{\mathbf{P}}_{k}^{H}\right\}^{-1} .
$$

Dong et al. [11] and Zheng and Xiao [12] uses LS based channel estimation to obtain $\hat{\mathbf{P}}_{k}^{\mathrm{LS}}$ and then derive the interpolation vector of WF-LS $\hat{\mathbf{C}}_{d, k}^{\mathrm{WF}-\mathrm{LS}}$, which completely neglects the effect of ICI. Therefore, the expression for WF-LS is expressed as $\hat{\mathbf{H}}_{d}^{\mathrm{WF}-\mathrm{LS}}(k)=\hat{\mathbf{C}}_{d, k}^{\mathrm{WF}-\mathrm{LS}} \hat{\mathbf{P}}_{k}^{\mathrm{LS}}$.
In this article, Channel and noise are assumed to be independent from each other and noise is complex Gaussian distribution with zero mean and $\sigma^{2}$ variance.

\section{Further analysis of WF-LS based channel estimation}

Least squares based channel estimate is given by

$$
\hat{\mathbf{H}}_{p_{i}}^{\mathrm{LS}}(k)=\mathbf{H}_{p_{i}}(k)+\frac{\mathbf{W}_{p_{i}}(k)}{\mathbf{X}_{p_{i}}(k)}
$$

where $\mathbf{W}_{p_{i}}=\left[\begin{array}{llll}W_{p_{i}}^{(0)} & W_{p_{i}}^{(1)} \ldots & W_{p_{i}}^{\left(N_{p}-1\right)}\end{array}\right]^{T}$ denotes additive complex Gaussian noise in the frequency domain. Substituting (3) into (2) and letting $M=2, \widehat{\mathbf{C}}_{d, k(2-O)}^{\mathrm{WF}-\mathrm{LS}}$ (with the subscript $m$ - $O$ representing WF-LS with orders $m$ ) can be expressed as

$$
\begin{aligned}
& \hat{\mathbf{C}}_{d, k(2-O)}^{\mathrm{WF}-\mathrm{LS}}(0)=\frac{B\left(d-p_{0}\right)\left(B(0)+\sigma^{2}\right)-B\left(d-p_{1}\right) B\left(p_{1}-p_{0}\right)}{D^{2-O}}, \\
& \hat{\mathbf{C}}_{d, k(2-O)}^{\mathrm{WF}-\mathrm{LS}}(1)=\frac{B\left(d-p_{1}\right)\left(B(0)+\sigma^{2}\right)-B\left(d-p_{0}\right) B\left(p_{0}-p_{1}\right)}{D^{2-O}},
\end{aligned}
$$

where $D^{2-O}=B^{2}(0)-B\left(p_{0}-p_{1}\right) B\left(p_{1}-p_{0}\right), D^{m-O}$ represents the corresponding determinant of WF-LS with orders $m$, and

$$
B\left(\Delta_{p}\right) \triangleq E\left\{\mathbf{H}_{p}(k) \mathbf{H}_{p+\Delta_{p}}^{*}(k)\right\}=J_{0}\left(2 \pi \Delta_{p} f_{D} T\right)=\sum_{l=0}^{\infty} \frac{\left(-\pi^{2} f_{D}^{2} T^{2} \Delta_{p}^{2}\right)^{l}}{l ! \Gamma(l+1)}
$$

is the auto-correlation function of the channel frequency response at the $k$-th subcarrier of different OFDM symbols [16], where $J_{0}(\cdot)$ is the zero-th order Bessel function of the first kind and $\Gamma(\cdot)$ is the gamma function. Meanwhile, the coefficients of linear interpolation is given by

$$
\begin{gathered}
\hat{\mathbf{C}}_{d, k}^{\text {Linear }}(0)=\frac{p_{1}-d}{p_{1}-p_{0}}, \\
\hat{\mathbf{C}}_{d, k}^{\text {Linear }}(1)=\frac{d-p_{0}}{p_{1}-p_{0}} .
\end{gathered}
$$

In the following, we will first show that linear interpolation is in fact a special case of WF-LS with orders 2, or put it another way, WF-LS with orders 2 will "degrade" into linear interpolation asymptotically. Then, we will analyze the asymptotical relationships among WF-LS with different orders. Finally we will point out the limitation of WF-LS. Let $l=1$ in (5) and substitute the resulting (5) into (4a) and (4b), after some manipulations we can obtain the expanded expression for $\hat{\mathbf{C}}_{d, k(2-O)}^{\mathrm{WF}-\mathrm{LS}}(i), i=0,1$ as 
$\hat{\mathrm{C}}_{d, k(2-O)}^{\mathrm{WF}(0)}(0)=$

$\frac{\left(1-\pi^{2} f_{D}^{2} T^{2}\left(d-p_{0}\right)^{2}\right) \sigma^{2}-\pi^{2} f_{D}^{2} T^{2}\left(-2 d p_{0}-2 p_{1}^{2}+2 p_{0} p_{1}+2 d p_{1}\right)-\pi^{4} f_{D}^{4} T^{4}\left(d-p_{1}\right)^{2}\left(p_{1}-p_{0}\right)^{2}}{2 \sigma^{2}+\sigma^{4}+2 \pi^{2} f_{D}{ }^{2}\left(p_{0}-p_{1}\right)^{2}-\pi^{4} f_{D}\left(p_{0}-p_{1}\right)}$

$\hat{\mathrm{C}}_{d, k(2-O)}^{\mathrm{WF}-\mathrm{L} S}(1)=$

$\frac{\left(1-\pi^{2} f_{D}^{2} T^{2}\left(d-p_{1}\right)^{2}\right) \sigma^{2}-\pi^{2} f_{D}^{2} T^{2}\left(-2 d p_{1}-2 p_{0}^{2}+2 p_{0} p_{1}+2 d p_{0}\right)-\pi^{4} f_{D}^{4} T^{4}\left(d-p_{0}\right)^{2}\left(p_{1}-p_{0}\right)^{2}}{2 \sigma^{2}+\sigma^{4}+2 \pi^{2} f_{2}^{2} T^{2}\left(p_{0}-p_{1}\right)^{2}-\pi^{4} f_{D}^{4} T^{4}\left(p_{0}-p_{1}\right)^{4}}$

Notice that (7a) and (7b) hold true when the wireless channel is static or in very slow moving environments. When $S N R \rightarrow \infty$, that is, when $\sigma^{2} \rightarrow 0$ (i.e., in the absence of noise), and inserting (6a) and (6b) into (7a) and (7b), respectively, then the relationship between the coefficients of WF-LS with orders 2 and that of linear interpolation can be obtained as

$$
\hat{\mathbf{C}}_{d, k(2-O)}^{\mathrm{WF}-\mathrm{LS}}(i)-\hat{\mathbf{C}}_{d, k}^{\mathrm{Linear}}(i)=\frac{\pi^{2} f_{D}^{2} T^{2}\left(d-p_{0}\right)\left(p-p_{1}\right)}{-2+\pi^{2} f_{D}^{2} T^{2}\left(p_{0}-p_{1}\right)^{2}}
$$

From (8) it is observed that as $f_{D} T \rightarrow 0$, the desired result

$$
\lim _{f_{D} T, \sigma^{2} \rightarrow 0} \hat{\mathbf{C}}_{d, k(2-O)}^{\mathrm{WF}-\mathrm{LS}}(i) \approx \hat{\mathbf{C}}_{d, k}^{\text {Linear }}(i), i=0,1
$$

can be established, as shown in Figure 2. It is worth noting that a totally different looking Figure 2 will be obtained if we choose another set of $p_{0}, p_{1}$ and $p$, but the asymptotical behavior as $f_{D} T \rightarrow 0$ will definitely be the same, as proved and explained in (8). Therefore, it can be inferred that WF-LS with orders 2 implicitly exists certain linearity which results in its failure in high speed communication environments, in which case the approximate linearity of the channel frequency response $\hat{\mathbf{H}}_{p_{0}}(k)$ and $\hat{\mathbf{H}}_{p_{1}}(k)$ between $p_{0}$ and $p_{1}$ disappears because of fast fading channels. On the other hand, because the channel estimation at pilot symbols is fixed to LS based channel estimation, if we want to improve the performance of WF-LS, one has to resort to WF-LS with higher orders (i.e., choosing $M>2$ in (2)) and hopefully that by adopting WF-LS with higher orders we can improve the estimation accuracy through using more pilot symbols, i.e., the MSE of channel estimation may be decreased by collecting more pilot symbols [17]. However, without properly adopting WF-LS with higher orders, there will be no performance improvement. In what follows we will investigate the asymptotical behavior of WF-LS with higher orders and show that WF-LS with higher orders can indeed be "degraded" into WF-LS with orders 2 if not carefully designed.

Substituting (3) and (5) into (2) and letting $M=3$ we can obtain $\hat{\mathbf{C}}_{d, k(3-O)}^{\mathrm{WF}-\mathrm{LS}}(0), \hat{\mathbf{C}}_{d, k(3-O)}^{\mathrm{WF}-\mathrm{LS}}(1)$ and $\hat{\mathbf{C}}_{d, k(3-O)}^{\mathrm{WF}-\mathrm{LS}}(2)$. Then, substituting (4a) and (4b) into the resulting
$\hat{\mathbf{C}}_{d, k(3-O)}^{\mathrm{WF}-\mathrm{LS}}(1)$ and $\hat{\mathbf{C}}_{d, k(3-O)}^{\mathrm{WF}-\mathrm{LS}}(1)$, respectively, after a few tedious, but otherwise straightforward, algebraic manipulations, we can obtain the relationships between $D^{3-O}$ and $D^{2-O}, \hat{\mathbf{C}}_{d, k(3-O)}^{\mathrm{WF}-\mathrm{LS}}(0)$ and $\hat{\mathbf{C}}_{d, k(2-O)}^{\mathrm{WF}-\mathrm{LS}}(0), \hat{\mathbf{C}}_{d, k(3-O)}^{\mathrm{WF}-\mathrm{LS}}(1)$ and $\hat{\mathbf{C}}_{d, k(2-O)}^{\mathrm{WF}-\mathrm{LS}}(1)$, and finally $\hat{\mathbf{C}}_{d, k(3-O)}^{\mathrm{WF}-\mathrm{LS}}(2)$ as

$$
\begin{gathered}
D^{3-O}=\left(D^{2-O}-B^{2}\left(p_{2}-p_{0}\right)-B^{2}\left(p_{2}-p_{1}\right)\right)\left(1+\sigma^{2}\right) \\
\quad+2 B\left(p_{2}-p_{0}\right) B\left(p_{2}-p_{1}\right) B\left(p_{1}-p_{0}\right), \\
\hat{\mathrm{C}}_{d, k(3-O)}^{\mathrm{WF}-\mathrm{LS}}(0)= \\
\frac{1}{D^{3-O}}\left[D^{2-O} \hat{\mathbf{C}}_{d, k(2-O)}^{\mathrm{WF}-\mathrm{LS}}(0)+B\left(d-p_{0}\right)\left(\sigma^{2}+\sigma^{4}\right)\right. \\
\quad-\left(B\left(d-p_{1}\right) B\left(p_{1}-p_{0}\right)+B\left(d-p_{2}\right) B\left(p_{2}-p_{0}\right)\right) \sigma^{2}- \\
B\left(d-p_{2}\right) B\left(p_{2}-p_{0}\right)-B\left(d-p_{0}\right) B^{2}\left(p_{2}-p_{1}\right) \\
\left.+B\left(d-p_{1}\right) B\left(p_{2}-p_{1}\right) B\left(p_{2}-p_{0}\right)+B\left(d-p_{2}\right) B\left(p_{1}-p_{0}\right) B\left(p_{2}-p_{1}\right)\right], \\
\hat{\mathrm{C}}_{d, k(3-O)}^{\mathrm{WF}-\mathrm{LS}}(1)= \\
\frac{1}{D^{3-O}}\left[D^{2-O} \hat{\mathbf{C}}_{d, k(2-O)}^{\mathrm{WF}-\mathrm{LS}}(1)+B\left(d-p_{1}\right)\left(\sigma^{2}+\sigma^{4}\right)\right. \\
\quad-\left(B\left(d-p_{0}\right) B\left(p_{1}-p_{0}\right)+B\left(d-p_{2}\right) B\left(p_{2}-p_{1}\right)\right) \sigma^{2}- \\
B\left(d-p_{2}\right) B\left(p_{2}-p_{1}\right)-B\left(d-p_{1}\right) B^{2}\left(p_{2}-p_{0}\right) \\
\left.+B\left(d-p_{0}\right) B\left(p_{2}-p_{0}\right) B\left(p_{2}-p_{1}\right)+B\left(d-p_{2}\right) B\left(p_{1}-p_{0}\right) B\left(p_{2}-p_{0}\right)\right], \\
\hat{\mathrm{C}}_{d, k(3-O)}^{\mathrm{WF}-\mathrm{LS})}(2)= \\
\frac{1}{D^{3-O}}\left[B\left(d-p_{2}\right)\left(1+\sigma^{2}\right)^{2}-\left(B\left(d-p_{0}\right) B\left(p_{0}-p_{2}\right)\right.\right. \\
\left.\quad+B\left(d-p_{1}\right) B\left(p_{1}-p_{2}\right)\right)\left(1+\sigma^{2}\right)+ \\
B\left(d-p_{0}\right) B\left(p_{1}-p_{0}\right) B\left(p_{2}-p_{1}\right)+B\left(d-p_{1}\right) B\left(p_{2}-p_{0}\right) B\left(p_{1}-p_{0}\right) \\
\left.\quad B\left(d-p_{2}\right) B^{2}\left(p_{1}-p_{0}\right)\right] .
\end{gathered}
$$

It is observed from (10) that as $\sigma^{2} \rightarrow 0$ and by fixing $p$, $p_{0}$ and $p_{1}$ (it is assumed here that we already have or know $p, p_{0}$ and $p_{1}$ for WF-LS with orders 2), what really matters are $f_{D} T$ and the distance between $p_{1}$ and $p_{2}$. By using (10) and putting different values of $f_{D} T$ and the distance between $p_{1}$ and $p_{2}$, we will obtain some interesting results as shown in Figure 3. It can be seen from Figure 3 that as $p_{2}$ increases, $\hat{\mathbf{C}}_{d, k(3-O)}^{\mathrm{WF}-\mathrm{LS}}(0)$ and $\hat{\mathbf{C}}_{d, k(2-O)}^{\mathrm{WF}-\mathrm{LS}}(0)$ will converge, and so will be $\hat{\mathbf{C}}_{d, k(3-O)}^{\mathrm{WF}-\mathrm{LS}}(1)$ and $\hat{\mathbf{C}}_{d, k(2-O)}^{\mathrm{WF}-\mathrm{LS}}(1)$. Meanwhile, $\hat{\mathbf{C}}_{d, k(3-O)}^{\mathrm{WF}-\mathrm{LS}}(2)$ will converge to zero as $p_{2}$ increases. On the other hand, this convergence will change with $f_{D} T$, as can also be observed from Figure 3. In this case, WF-LS with orders 3 will "degrade" into WF-LS with orders 2, which means that even more pilot symbols are adopted to estimate $\hat{\mathbf{H}}_{d}^{\mathrm{WF}-\mathrm{LS}}(k)$, there will be no performance gains at all. Also note here that as in Figure 2 , if we choose another set of $p_{0}, p_{1}, p_{2}$ and $p$ we will obtain a different looking Figure 3, but the asymptotical convergence behavior will be the same. This asymptotical convergence behavior can be explained by investigating (10), which shows that $B\left(\Delta_{p}\right)$ will become small and close to zero as $f_{D} T\left(p_{2}-p_{i}\right), i=0,1$ grows large and tends to infinity. In conclusion we have the following results in the absence of noise 


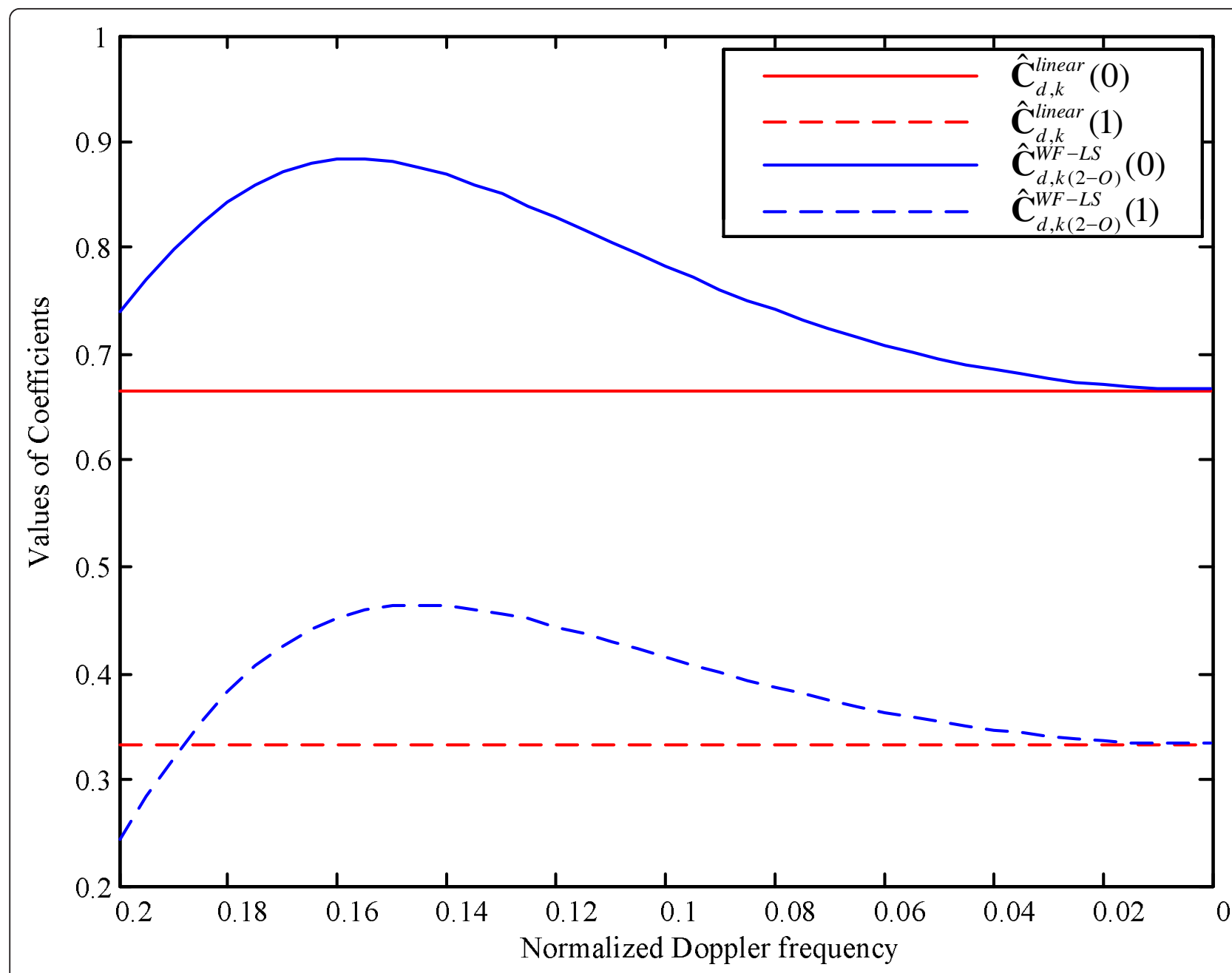

Figure 2 The relationship between the coefficients of Wiener filter with orders 2 and that of linear interpolation. In this figure we let $\sigma^{2}$ $\rightarrow 0, p_{0}=1, p_{1}=4$ and $p=2$.

$$
\begin{aligned}
& \lim _{\substack{p_{2} \rightarrow \infty, \sigma^{2} \rightarrow 0 \\
\text { affected by } f_{D} T}} \hat{\mathbf{C}}_{d, k(3-O)}^{\mathrm{WF}-\mathrm{LS}}(i) \approx \hat{\mathbf{C}}_{d, k(2-O)}^{\mathrm{WF}-\mathrm{LS}}(i), \quad i=0,1 \\
& \lim _{\substack{p_{2} \rightarrow \infty, \sigma^{2} \rightarrow 0 \\
\text { affected by } f_{\mathrm{D}} T}} \hat{\mathbf{C}}_{d, k(3-O)}^{\mathrm{WF}-\mathrm{LS}}(i) \approx 0, \quad i=2 .
\end{aligned}
$$

When noise is present, the convergence behavior can be obtained through similar analysis by investigating (10) and the result is shown in Figure 4. This idea of analyzing the relationship between WF-LS with orders 3 and WF-LS with orders 2 can be further extended to analyze the relationships among WF-LS with different orders, which can be inferred directly from the above analysis that when $p_{m}$ is sufficiently large (for $m>2$ )

$$
\begin{array}{rlrl}
\hat{\mathrm{C}}_{d, k((m+1)-O)}^{\mathrm{WF}-\mathrm{LS}}(i) \approx \hat{\mathrm{C}}_{d, k(m-O)}^{\mathrm{WF}-\mathrm{LS}}(i), & & i=0,1, \ldots, m-1 \\
\hat{\mathrm{C}}_{d, k((m+1)-O)}^{\mathrm{WF} \mathrm{L}}(i) \approx 0, & i=m .
\end{array}
$$

Therefore we conclude that we should carefully avoid these "convergence points" when we are trying to improve the performance of WF-LS with low orders by using WF-LS with higher orders, otherwise this goodwill will prove to be in vain. Of course, we can choose $p_{m}$ arbitrarily close to $p_{m-1}$, but this will cause transmitting more pilot symbols, which will significantly reduce the spectral efficiency. However, we can not choose $p_{m}$ arbitrarily large to increase the spectral efficiency, as have been proved that this will result in no performance improvement. Meanwhile the choose of $p_{m}$ is related to $f_{D} T$. For example, we observe that when $\mathrm{SNR}=30 \mathrm{~dB}$ and $f_{\mathrm{D}} T=0.01$, in order for $\hat{\mathbf{C}}_{d, k(3-O)}^{\mathrm{WF}-\mathrm{LS}}(0), \hat{\mathbf{C}}_{d, k(3-O)}^{\mathrm{WF}-\mathrm{LS}}(1)$ and $\hat{\mathbf{C}}_{d, k(3-O)}^{\mathrm{WF}-\mathrm{LS}}(2)$ to have a negligible difference between $\hat{\mathbf{C}}_{d, k(2-O)}^{\mathrm{WF}-\mathrm{LS}}(1), \hat{\mathbf{C}}_{d, k(2-O)}^{\mathrm{WF}-\mathrm{LS}}(1)$ and 0 , respectively, $p_{2}$ should 


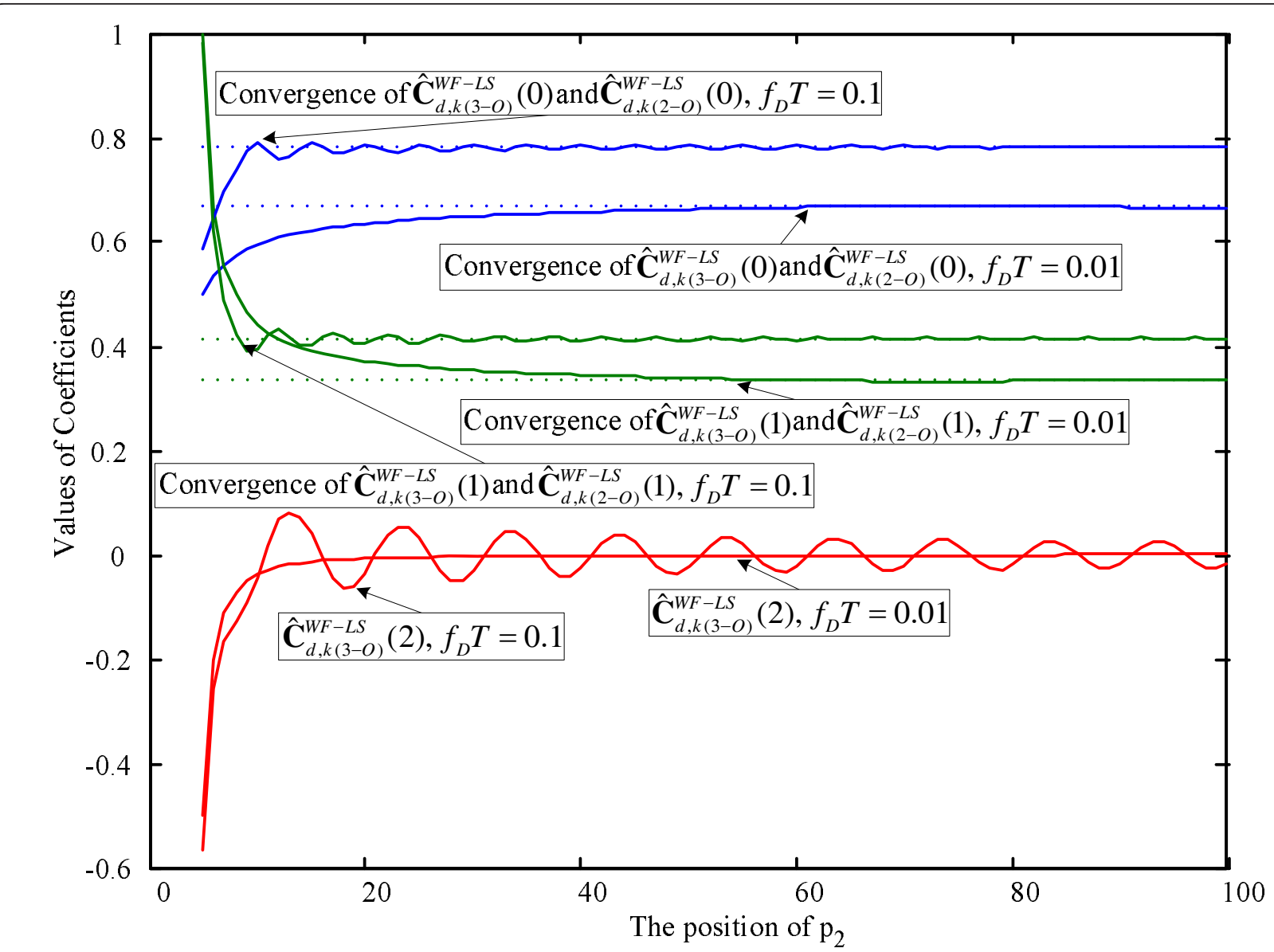

Figure 3 The relationship between the coefficients of Wiener filter with orders 3 and that of Wiener filter with orders 2 . In this figure we let $\sigma^{2} \rightarrow 0, p_{0}=1, p_{1}=4$ and $p=2$. The dashed lines represents the coefficients of Wiener filter with orders 2 .

be bigger than about 54 and 56. When $\mathrm{SNR}=30 \mathrm{~dB}$ and $f_{D} T=0.1$, for $\hat{\mathbf{C}}_{d, k(3-O)}^{\mathrm{WF}-\mathrm{LS}}(0), \hat{\mathbf{C}}_{d, k(3-O)}^{\mathrm{WF}-\mathrm{LS}}(1)$ and $\hat{\mathbf{C}}_{d, k(3-O)}^{\mathrm{WF}-\mathrm{LS}}(2)$ to have a negligible difference between $\hat{\mathbf{C}}_{d, k(2-O)}^{\mathrm{WF}-\mathrm{LS}}(1), \hat{\mathbf{C}}_{d, k(2-O)}^{\mathrm{WF}-\mathrm{LS}}(1)$ and 0 , respectively, $p_{2}$ should be bigger than 9 . The above observations are also approximately true for other SNR values. Therefore, from the above observations and analysis we may conclude that the combined parameter $f_{D} T\left(p_{m}-p_{m-1}\right)$ should be smaller than about 0.5 to avoid the degradation of WF with higher orders. This result is coincide with the sampling theorem in the time domain [18], which is, $f_{D} T\left(p_{m}-p_{m-1}\right)<0.5$. Till this point we can say that we have just proved the sampling theorem in the time domain, from a new perspective, i.e., from the coefficients of WF-LS point of view. The optimal value of $f_{D} T\left(p_{m}-p_{m-1}\right)$ that will simultaneously increase the performance while maintaining the spectral efficiency is dependent on the specific problem at hand and related to the pilot symbol and system design problems, which is beyond the scope of this article, but will be an interesting and a meaningful topic for the future work.

Although the performance of WF-LS can be improved by adopting more pilot symbols provided that the above conditions are satisfied, WF-LS has an intrinsic weakness that will lead to its decreased performance in high speed mobile environments. As will be seen in Section 5 , the performance of WF-LS is acceptable in slow to moderate mobile environments, but decreases significantly in high speed environments. This degradation of WF-LS is due mainly to the fatal weakness of the underlining assumption of LS based channel estimation that the channel coefficients within one OFDM symbol period are constants. This assumption will generally not hold true in time-varying channels, especially in fast time-varying cases. In light of the above analysis and considering that BEM based channel estimation method $[13,14]$ can accurately estimate the time-varying channel coefficients of an OFDM symbol, we propose to combine BEM based channel estimation with WF to deal with channel estimation in time-varying channels. 


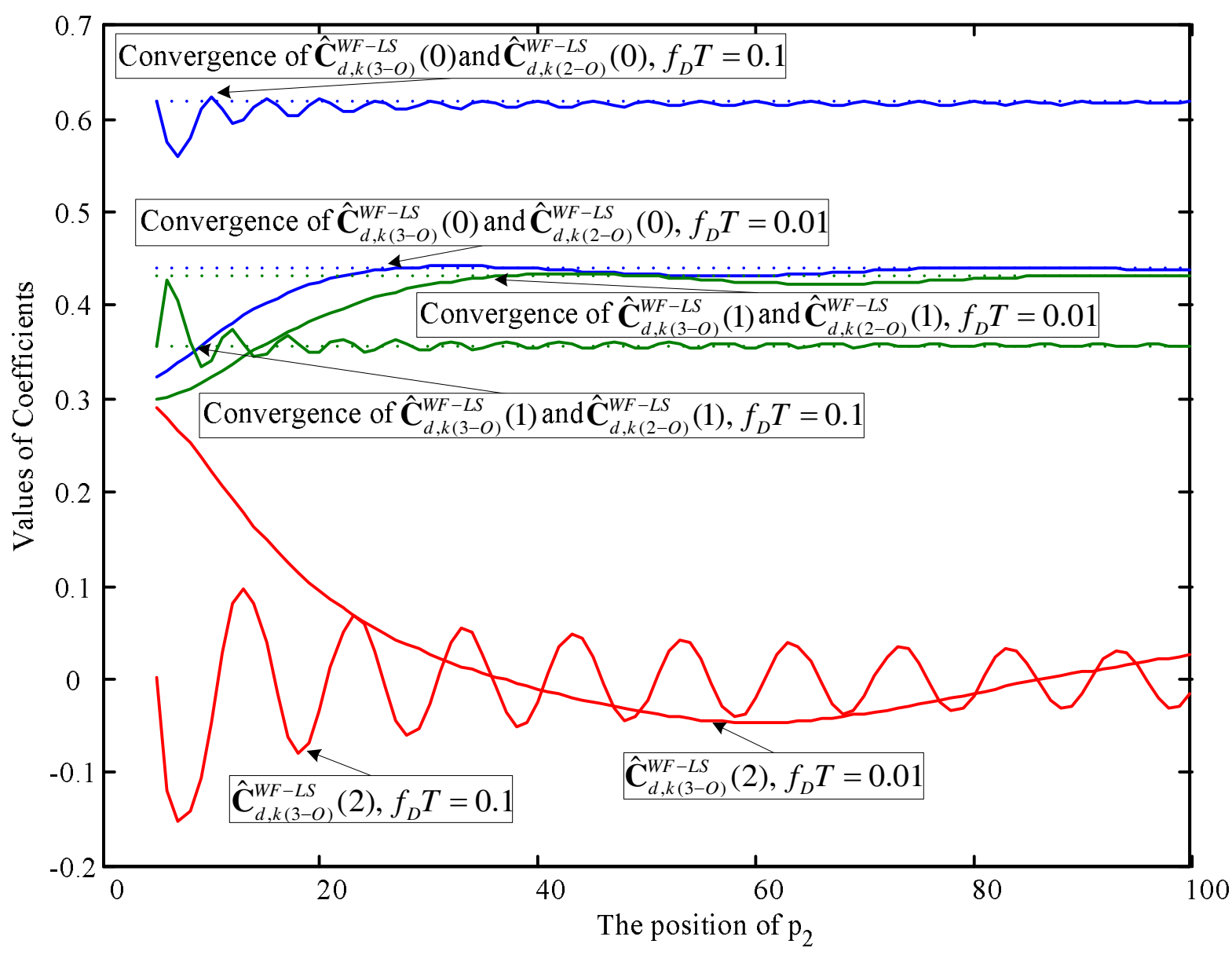

Figure 4 The relationship between the coefficients of Wiener filter with orders 3 and that of Wiener filter with orders 2 . In this figure we let $S N R \rightarrow 0, p_{0}=1, p_{1}=4$ and $p=2$. The dashed lines represents the coefficients of Wiener filter with orders 2 .

4 The expression for the proposed WF-BEM based channel estimation

Basis expansion model based channel impulse response of the $p_{i}$-th pilot symbol can be expressed as $[13,14]$

$$
h_{p_{i}}(n, l)=\sum_{q=0}^{Q} \eta_{q, l}\left(p_{i}\right) b_{n, q}
$$

where $h_{p_{i}}(n, l)$ represents the channel impulse response of the $l$-th path at time $n$ within the $p_{i}$-th pilot symbol period and is assumed to be modeled as a wide sense stationary (WSS) complex Gaussian process with a statistically independent path, $n=0,1, \ldots, N$ $1, N$ is the symbol length $\left(N_{p} \leq N\right), l=0,1, \ldots, L-1$, $L$ is the length of wireless channel, $\eta_{q, l}\left(p_{i}\right)$ is the coefficient of BEM, $b_{n, q}$ is the base that captures channel time variations, and $Q$ is the number of BEM bases. BEM is motivated by the observation that the temporal (n) variation of $h(n, l)$ is usually rather smooth due to the channel's limited Doppler spread, and therefore, $\left\{b_{n, q}\right\}_{q=0}^{Q}$ can be chosen as a small set (i.e., $\left.Q \ll N\right)$ of smooth functions.

For the pilot subcarriers in the $p_{i}$-th pilot symbol, an equation consisting of $\eta_{q, l}\left(p_{i}\right)$ can be expressed as [14]

$$
\mathbf{Y}_{p_{i}}=\mathbf{P} \eta_{p_{i}}+Z_{p_{i}}
$$

where $\mathbf{Y}_{p_{i}}=\left[Y_{p_{i}}^{(0)} Y_{p_{i}}^{(1)} \ldots Y_{p_{i}}^{\left(N_{p}-1\right)}\right]^{T}$ is the received signal at the pilot subcarriers of the $p_{i}$-th pilot symbol in the frequency domain, $\eta_{p_{i}}=\left[\eta_{0,0}\left(p_{i}\right), \ldots, \eta_{0, L-1}\left(p_{i}\right), \ldots, \eta_{Q, 0}\left(p_{i}\right), \ldots, \eta_{Q, L-1}\left(p_{i}\right)\right]^{T}$ represents the coefficients of BEM to be estimated, $\mathbf{P}$ consists of the base, the pilot subcarriers and the Fourier transform matrix at the positions of according pilot subcarriers [14, Eq. (14)], and $\boldsymbol{Z}_{p_{i}}$ is the interference from data subcarriers and noise. In this article, the regularized LS method [19] is adopted to estimate $\boldsymbol{\eta}_{p_{i}}$ as

$$
\hat{\boldsymbol{\eta}}_{p_{i}}=\left(\mathbf{P}^{H} \mathbf{P}+a \mathbf{I}\right)^{-1} \mathbf{P}^{H} \mathbf{Y}_{p_{i}}
$$


where small value of $a$ is to ensure the matrix $\mathbf{P}^{H} \mathbf{P}+$ $a \mathbf{I}$ a matrix with full rank. Let us define a matrix $\mathbf{A} \triangleq$ $\left(\mathbf{P}^{H} \mathbf{P}+a \mathbf{I}\right)^{-1} \mathbf{P}^{H}$ in (15) and $\mathbf{A}$ can be further divided into $Q+1$ sub-matrices, each of which is of size $L \times$ $N_{p}$. More specifically, A can be expressed as

$$
\mathbf{A}=\left[\left[\mathbf{A}_{q=0}\right]_{L \times N_{p}}^{T}\left[\mathbf{A}_{q=1}\right]_{L \times N_{p}}^{T} \ldots\left[\mathbf{A}_{q=Q}\right]_{L \times N_{p}}^{T}\right]_{(Q+1) L \times N_{p}}^{T} .
$$

Combing (15) and (16), $\hat{\eta}_{q, l}\left(p_{i}\right)$ can be expressed as

$$
\hat{\eta}_{q, l}\left(p_{i}\right)=\sum_{k=0}^{N_{p}-1} \mathbf{A}_{q}(l, k) \mathbf{Y}_{p_{i}}(k)
$$

As shown in [20], in various mobile environments, the received signal $\mathbf{Y}_{p_{i}}(k)$ at the $k$-th subcarrier is expressed as

$$
\mathbf{Y}_{p_{i}}(k)=\mathbf{H}_{p_{i}}(k) \mathbf{X}_{p_{i}}(k)+\underbrace{\sum_{\substack{z=0 \\ z \neq k}}^{N-1} \mathbf{H}_{p_{i}}^{\mathrm{IC} 1}(k, z) \mathbf{X}_{p_{i}}(z)}_{\text {ICI term }}+\mathbf{W}_{p_{i}}(k),
$$

where $\mathbf{W}_{p_{i}}(k)$ is an additive complex Gaussian noise in the frequency domain,

$$
\mathbf{H}_{p_{i}}(k)=\frac{1}{N} \sum_{l=0}^{L-1} \sum_{n=0}^{N-1} h_{p_{i}}(n, l) e^{\frac{-j 2 \pi k l}{N}}
$$

represents the channel frequency response corresponding to the desired subcarrier $k$ and

$$
\mathbf{H}_{p_{i}}^{\mathrm{IC} 1}(k, z)=\frac{1}{N} \sum_{l=0}^{L-1} \sum_{n=0}^{N-1} h_{p_{i}}(n, l) e^{\frac{-j 2 \pi z l}{N}} e \frac{-j 2 \pi(k-z) n}{N}(20)
$$

is the ICI coefficient, which is the the off-diagonal element in the channel frequency response matrix. Since the ICI term consists of a large number of random interferences and is based on the assumption that $h_{p_{i}}(n, l)$ is WSS complex Gaussian process, we can model the term as additive white Gaussian noise according to the central limit theorem [21].

To derive the expression for WF-BEM that considers the effect of ICI, the following steps are proposed:

- Step (1) Substitute (17) into (13). We can get the estimate of BEM based channel impulse response as

$$
\hat{h}_{p_{i}}(n, l)=\sum_{q=0}^{Q} \sum_{k=0}^{N_{p}-1} \mathbf{A}_{q}(l, k) \mathbf{Y}_{p_{i}}(k) b_{n, q}
$$

- Step (2) Substitute (21) into (19). Then we can obtain the estimate of BEM based channel estimation $\hat{\mathbf{P}}_{k}^{\text {BEM }}$ with each element given by

$\hat{\mathbf{H}}_{p_{i}}^{\mathrm{BEM}}(k)=\frac{1}{N} \sum_{l=0}^{L-1} \sum_{n=0}^{N-1} \sum_{q=0}^{Q} \sum_{s=0}^{N_{p}-1} \mathbf{A}_{q}(l, s) \mathbf{Y}_{p_{i}}(s) b_{n, q} e^{\frac{-j 2 \pi k l}{N}}$.

- Step (3) Substitute (18) into (22). Hence, the relationships among $\hat{\mathbf{H}}_{p_{i}}^{\mathrm{BEM}}(k), \mathbf{H}_{p_{i}}(k)$ and the ICI term can be established. Therefore, $E\left\{\mathbf{H}_{d}(k)\left(\hat{\mathbf{H}}_{p_{i}}^{\mathrm{BEM}}(k)\right)^{*}\right\}$ can be derived as

$E\left\{\mathbf{H}_{d}(k)\left(\hat{\mathbf{H}}_{p_{i}}^{\mathrm{BEM}}(k)\right)^{*}\right\}=J_{0}\left(2 \pi\left(d-p_{i}\right) f_{\mathrm{D}} T\right)$

$$
\underbrace{\times \frac{1}{N} \sum_{l=0}^{L-1} \sum_{n=0}^{N-1} \sum_{q=0}^{Q} \sum_{s=0}^{N_{p}-1} \sum_{u=0}^{L-1} \mathbf{A}_{q}^{*}(l, s) \mathbf{X}_{p_{i}}^{*}(s) b_{n, q}^{*} \sigma_{u}^{2} e^{\frac{j 2 \pi(s u+k(l-u))}{N}} .}_{\beta_{p_{i}}(k)} .
$$

- Step (4) Use (20) to derive the correlation function of $\mathbf{H}_{p_{i}}^{\mathrm{IC} 1}(k, v)$ and $\mathbf{H}_{p_{i}}^{\mathrm{IC} 1}(k, z)$ as

$$
\begin{aligned}
E\left\{\mathbf{H}_{p_{i}}^{\mathrm{IC} 1}(k, v)\left(\mathbf{H}_{p_{i}}^{\mathrm{IC} 1}(k, z)\right)^{*}\right\}=\frac{1}{N^{2}} \sum_{n_{1}=0}^{N-1} \sum_{n_{2}=0}^{N-1} \sum_{l=0}^{L-1} \sigma_{l}^{2} \\
\quad \times J_{0}\left(2 \pi\left(n_{1}-n_{2}\right) f_{D} T_{s}\right) \\
\\
e^{\frac{-j 2 \pi\left((v-z) l+\left((k-v) n_{1}-(k-z) n_{2}\right)\right)}{N}} .
\end{aligned}
$$

Through the proposed Steps (1)-(4) and by using (22) and (24), $E\left\{\hat{\mathbf{H}}_{p_{i}}^{\mathrm{BEM}}(k)\left(\hat{\mathbf{H}}_{p_{j}}^{\mathrm{BEM}}(k)\right)^{*}\right\}$ can be derived as follows:

$$
\begin{aligned}
& \text { if } p_{i} \neq p_{j} \text {, } \\
& E\left\{\hat{\mathrm{H}}_{p_{i}}^{\mathrm{BEM}}(k)\left(\hat{\mathrm{H}}_{p_{j}}^{\mathrm{BEM}}(k)\right)^{*}\right\}=J_{0}\left(2 \pi\left(p_{i}-p_{j}\right) f_{\mathrm{D}} T\right) \\
& \times \underbrace{\left[\frac{1}{N^{2}} \sum_{l=0}^{L-1} \sum_{n=0}^{N-1} \sum_{m=0}^{N-1} \sum_{q=0}^{Q} \sum_{s=0}^{Q} \sum_{g=0}^{N_{p}-1} \sum_{r=0}^{N_{p}-1} \sum_{u=0}^{L-1} \mathbf{A}_{q}(l, g) \mathbf{A}_{s}^{*}(l, r) \mathbf{X}_{p_{i}}(g) \mathbf{X}_{p_{j}}^{*}(r)\left(b_{n, q} b_{m, s}^{*}\right) \sigma_{u}^{2} e^{\left.\frac{-j 2 \pi(g-r) u}{N}\right]}\right.}_{\phi_{\phi_{\varphi_{p}}}}
\end{aligned}
$$

and if $p_{i}=p_{j}$

$E\left(\hat{\mathbf{H}}_{p_{i}}^{\mathrm{BEM}}(k)\left(\hat{\mathrm{H}}_{p_{i}}^{\mathrm{BEM}}(k)\right)^{*}\right\} \triangleq \varphi_{p_{i}}=$

$\frac{1}{N^{2}} \sum_{l=0}^{L-1} \sum_{n=0}^{N-1} \sum_{m=0}^{N-1} \sum_{q=0}^{Q} \sum_{s=0}^{Q} \sum_{g=0}^{N_{p}-1} \sum_{r=0}^{N_{p}-1} \mathbf{A}_{q}(l, g) \mathbf{A}_{s}^{*}(l, r)\left(b_{n, q}, b_{m, s}^{*}\right)$

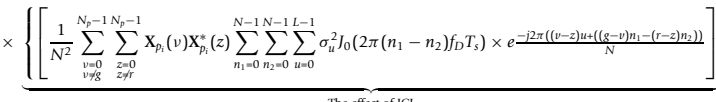

$$
\begin{aligned}
& +\left\{\begin{array}{c}
\text { The effect of ICI } \\
\left.1+\sigma^{2}\right\}, g=r_{,} \\
\left.\mathbf{X}_{p_{1}}(g) \mathbf{X}_{p_{1}}^{*}(r) \sum_{u_{1}=0}^{L-1} \sigma_{u_{1}}^{2} \frac{-j 2 \pi(g-r) u_{1}}{N}\right\}, g \neq r,
\end{array}\right.
\end{aligned}
$$

where we have used the fact that $E\left\{h_{p_{i}}\left(n_{1}, l\right) h_{p_{j}}^{*}\left(n_{2}, l\right)\right\}=\sigma_{l}^{2} J_{0}\left(2 \pi f_{D}\left(\left(p_{i}-p_{j}\right) T+\left(n_{1}-n_{2}\right) T_{s}\right)\right)$ and its corresponding function in the frequency domain, 
$\sigma_{l}^{2}$ is the power of the $l$-th path and without loss of generality we assume $\sum_{l=0}^{L-1} \sigma_{l}^{2}=1, T_{s}$ is the sampling period. It is noted that based on the assumption that $h_{p_{i}}(n, l)$ within a single OFDM symbol period are constants, the effect of ICI is neglected (i.e., in (20) $\left.\mathbf{H}_{p_{i}}^{\mathrm{ICI}}(k, z) \equiv 0\right)$, the authors Dong et al. [11] and Zheng and Xiao [12] only consider the noise variance $\sigma^{2}$. However, we consider the variation of $h_{p_{i}}(n, l)$ within a single OFDM symbol period in time-varying channels and our derived result explicitly considers both the noise variance and the effect of ICI, which can provide us with details about channel variations in various mobile environments.

- Step (5) Substitute (23), (25) and (26) into (2). Then, the interpolation vector of WF-BEM can be obtained as

$\hat{\mathrm{C}}_{d, k}^{\mathrm{WF} \cdot \mathrm{BEM}}=\left[J_{0}\left(2 \pi\left(d-p_{0}\right) f_{D} T\right) \beta_{p_{0}}(k) J_{0}\left(2 \pi\left(d-p_{1}\right) f_{D} T\right) \beta_{p_{1}}(k) \ldots J_{0}\left(2 \pi\left(d-p_{M-1}\right) f_{D} T\right) \beta_{P_{M-1}}(k)\right]$.

$\left[\begin{array}{cccc}\varphi_{p_{0}} & J_{0}\left(2 \pi\left(p_{0}-p_{1}\right) f_{D} T\right) \phi_{p_{0}, p_{1}} & \cdots J_{0}\left(2 \pi\left(p_{0}-p_{M-1}\right) f_{D} T\right) \phi_{p_{0}, p_{M-1}} \\ J_{0}\left(2 \pi\left(p_{1}-p_{0}\right) f_{D} T\right) \phi_{p_{1}, p_{0}} & \varphi_{p_{1} 1} & \cdots & J_{0}\left(2 \pi\left(p_{1}-p_{M-1}\right) f_{D} T\right) \phi_{p_{1}, p_{M-1}} \\ \vdots & \vdots & \ddots & \vdots \\ J_{0}\left(2 \pi\left(p_{M-1}-p_{0}\right) f_{D} T\right) \phi_{p_{M-1}, p_{0}} J_{0}\left(2 \pi\left(p_{M-1}-p_{1}\right) f_{D} T\right) \phi_{p_{M-1}, p_{1}} & \cdots & \varphi_{p_{M-1}}\end{array}\right](27)$

Comparing (27) with the interpolation vector of WFLS $\hat{\mathbf{C}}_{d, k}^{\mathrm{WF} \text { - LS }}$ given in [11, Eq. (29-31)] and [12, Eq. (29)], it can be seen that the coefficients $\beta_{p_{i}}(k), \phi_{p_{i}, p_{c}}$ and $\varphi_{p_{i}}$ are related with channel variations (for both the base $b_{n}$, $q$ and the effect of ICI) and unique to WF-BEM.

- Step (6) Multiply the estimate of BEM based channel estimation $\hat{\mathbf{P}}_{k}^{\text {BEM }}$ obtained in Step (2) by the interpolation vector of WF-BEM $\hat{\mathbf{C}}_{d, k}^{\mathrm{WF}-\mathrm{BEM}}$ obtained in Step (5). Finally, the expression for WF-BEM is given by

$$
\hat{\mathbf{H}}_{d}^{\mathrm{WF}-\mathrm{BEM}}(k)=\hat{\mathbf{C}}_{d, k}^{\mathrm{WF}-\mathrm{BEM}} \hat{\mathbf{P}}_{k}^{\mathrm{BEM}} .
$$

The proposed algorithm is summarized as follows. First, we use expression (22) to obtain the channel estimate of pilot symbols (corresponding to the Step 1 in Figure 1); then, we use expression (28) to obtain the channel estimate of data symbols (corresponding to the Step 2 in Figure 1).

In this section the interpolation vector of WF-BEM at the frequency dimension is not considered for the reason that by using BEM based channel estimation, the channel impulse response at a pilot symbol can be obtained by expression (21). Then, through transferring the channel impulse response into frequency domain by expression (22), the estimate of channel frequency response at required subcarriers of the pilot symbol can be obtained.

\section{Simulation results and discussions}

To evaluate the performance of the proposed WF-BEM algorithm, extensive computer simulations are carried out. LTE uplink system $[15,22]$ is considered in the simulation. The simulation parameters are listed in Table 1 and the generalized complex exponential BEM (GCE-BEM) [14] is adopted.

\subsection{MSE comparison}

The MSE curve of BEM based channel estimation with LS based channel estimation only at pilot symbols is shown in Figure 5. From Figure 5 it can be seen that in time-varying channels, BEM based channel estimation performs consistently better than LS based channel estimation. The estimation accuracy of BEM based channel estimation is much better than that of LS based channel estimation especially in high-speed mobile environment, as can be seen from Figure 5 . This result proves our analysis in Section 3 that in time-varying channels, as the assumption that the channel coefficients within one OFDM symbol period are constants no longer holds true, BEM based channel estimation will outperform LS based channel estimation. After interpolation along the time dimension by using WF, the MSE curve of WF-BEM with WF-LS is shown in Figure 6. It is seen from Figure 6 that due to the precondition that BEM based channel estimation is better than LS based channel estimation in time-varying channels, the estimation accuracy of the proposed WF-BEM is better than WF-LS.

As stated in Section 1, the channel estimation accuracy at data symbols is determined by both the channel estimation accuracy at pilot symbols and the accuracy of the interpolation method. WF is the best interpolation method at the time dimension to estimate channel at data symbols in terms of minimizing MSE. The optimality of WF is independent of the channel estimation method adopted at pilot symbols. Therefore we can

\section{Table 1 Simulation parameters}

\begin{tabular}{ll}
\hline Types of modulation & 16QAM and 64QAM \\
\hline Velocity & $30,120,350$ and $480 \mathrm{~km} / \mathrm{h}$ \\
Transmission bandwidth & $20 \mathrm{MHz}$ \\
Transmission block & 50 resource blocks \\
Carrier frequency & $2.3 \mathrm{GHz}$ \\
Channel model & Rayleigh fading \\
Doppler spectrum & Jake's model \\
Transmit and receive antenna & One transmit antenna and two receive \\
numbers & antennas \\
\hline
\end{tabular}




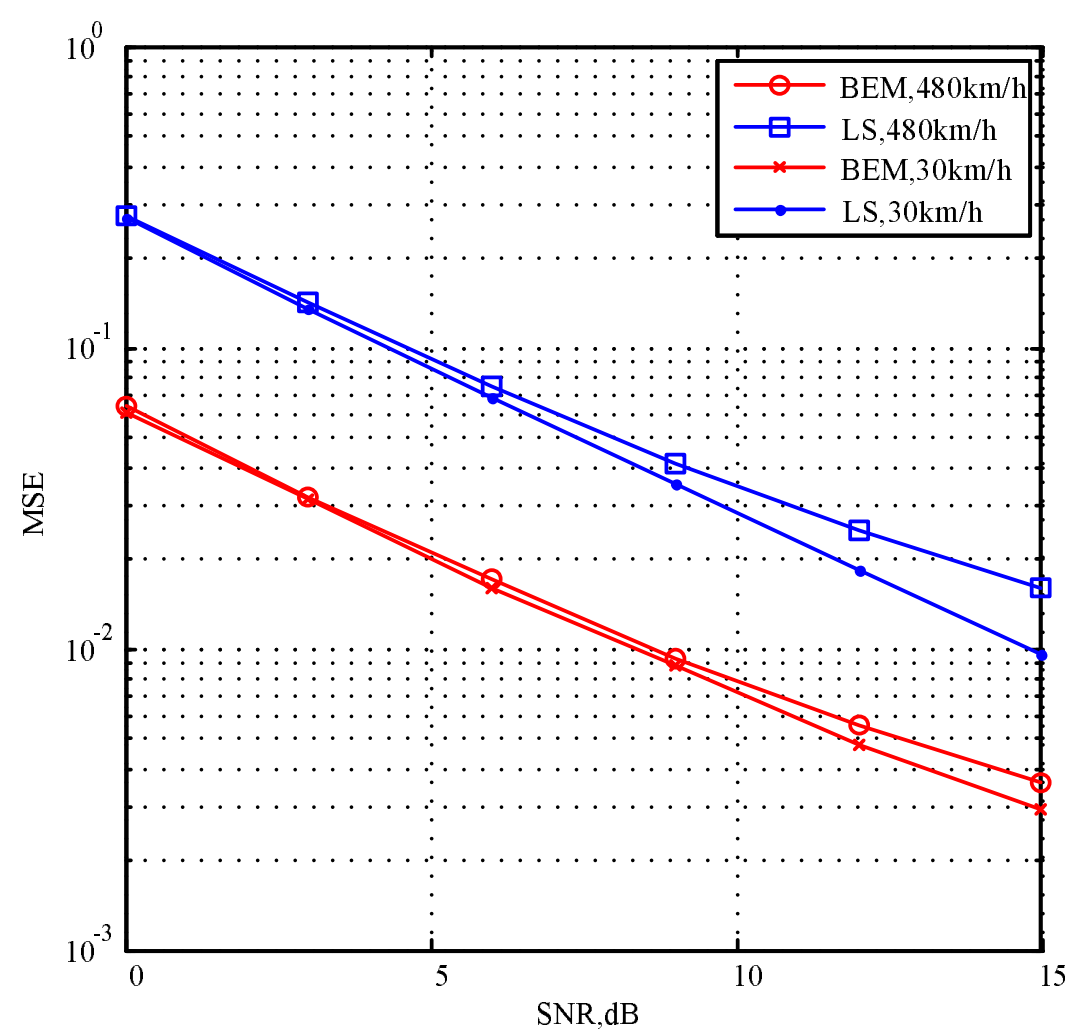

Figure 5 Performance of MSE only at pilot symbols. The blue lines represent the MSE of LS based channel estimation and the red lines represent that of BEM based channel estimation.

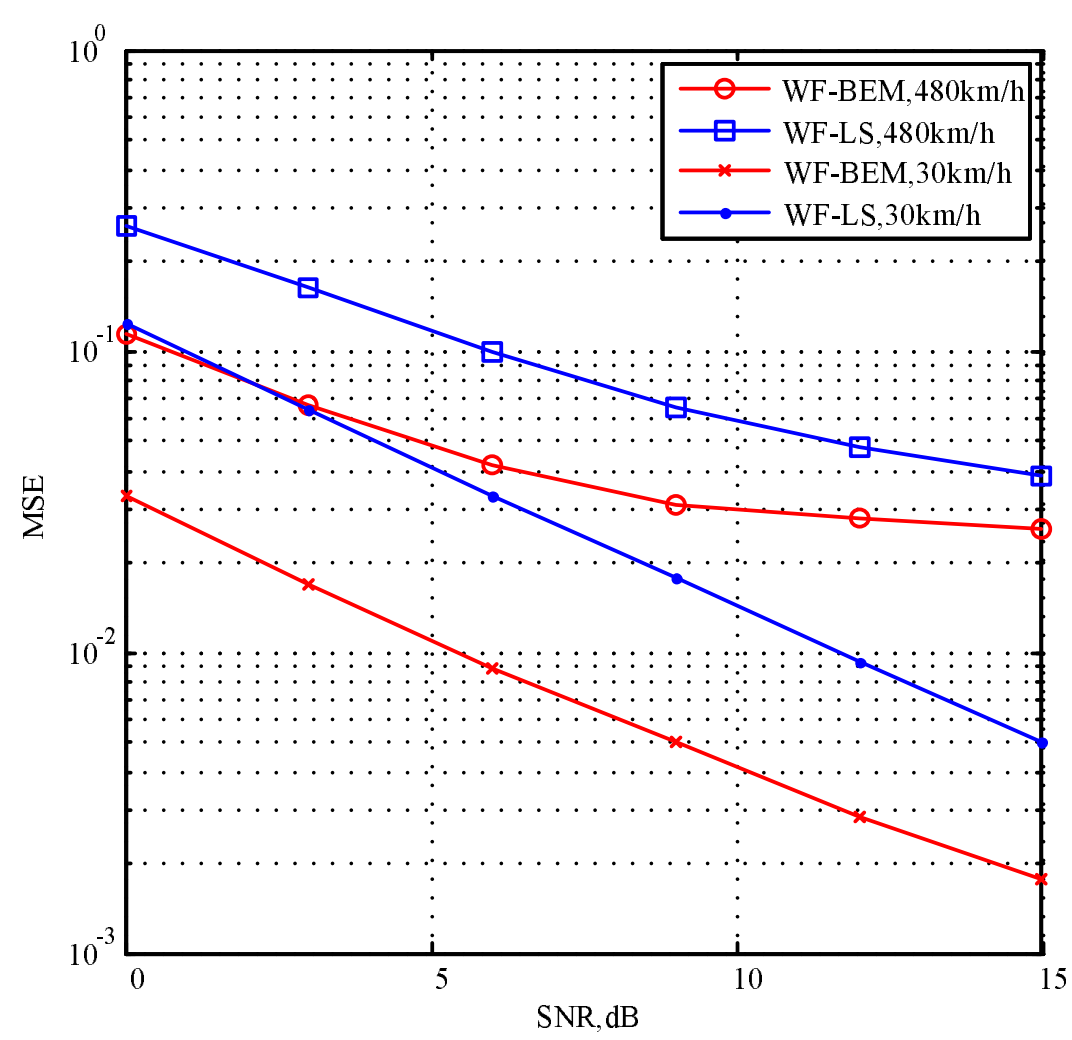

Figure 6 Performance of MSE after interpolation by Wiener filter. The blue lines represent the MSE of WF-LS and the red lines represent that of WF-BEM. 
infer that the reason that WF-LS underperforms is because the channel estimation accuracy of LS based channel estimation is worse than that of BEM based channel estimation at pilot symbols in time-varying channels, which have been proved by Figure 5 . This is also the main reason for our motivation to combine BEM based channel estimation with WF and derive the expression for WF-BEM based channel estimation to deal with channel estimation in time-varying channels.

Besides BEM based channel estimation can more accurately estimate fast time-varying channels than LS based channel estimation does, another reason that a channel estimator with higher accuracy results in improved performance in time-varying channels is illustrated as follows. In OFDM systems, Doppler effects and instabilities of the transmitter and receiver carrier frequency oscillators will cause a loss of orthogonality between the subcarriers, resulting in ICI. The carrier frequency offset caused by Doppler effects in time-varying channels is a fraction of the subcarrier spacing (i.e., in most practical cases the normalized Doppler frequency is less than 0.2). If we want to compensate this effect, we are dealing with the problem of fine-frequency adjustment. One effect of carrier frequency offset is the detrimental effect of a rotation of the subcarriers $[23,24]$. This effect will be recognized by a channel estimator, which does not distinguish between phase offsets caused by the channel and those caused by a frequency offset. Thus, a channel equalizer appears also to have fine frequency synchronization capabilities. Therefore it can be inferred that a channel estimator with higher accuracy can more accurately compensate this rotation caused by Doppler effects and thus will perform better in time-varying channels.

\subsection{BLER comparison}

Figures 7, 8, 9 and 10 report the block error rate (BLER) curve of WF-BEM with WF-LS, at the mobile speed of $30 \mathrm{~km} / \mathrm{h}$ (corresponding to the downtown environment), $120 \mathrm{~km} / \mathrm{h}$ (corresponding to the highway environment), 350 and $480 \mathrm{~km} / \mathrm{h}$ (corresponding to the high-speed train environments) respectively. Figure 7 illustrates that since the wireless channel can be regarded as nearly invariant during one OFDM symbol period at $30 \mathrm{~km} / \mathrm{h}$ and also because the Doppler frequency is small in this situation, the performance of WF-LS is close to that of

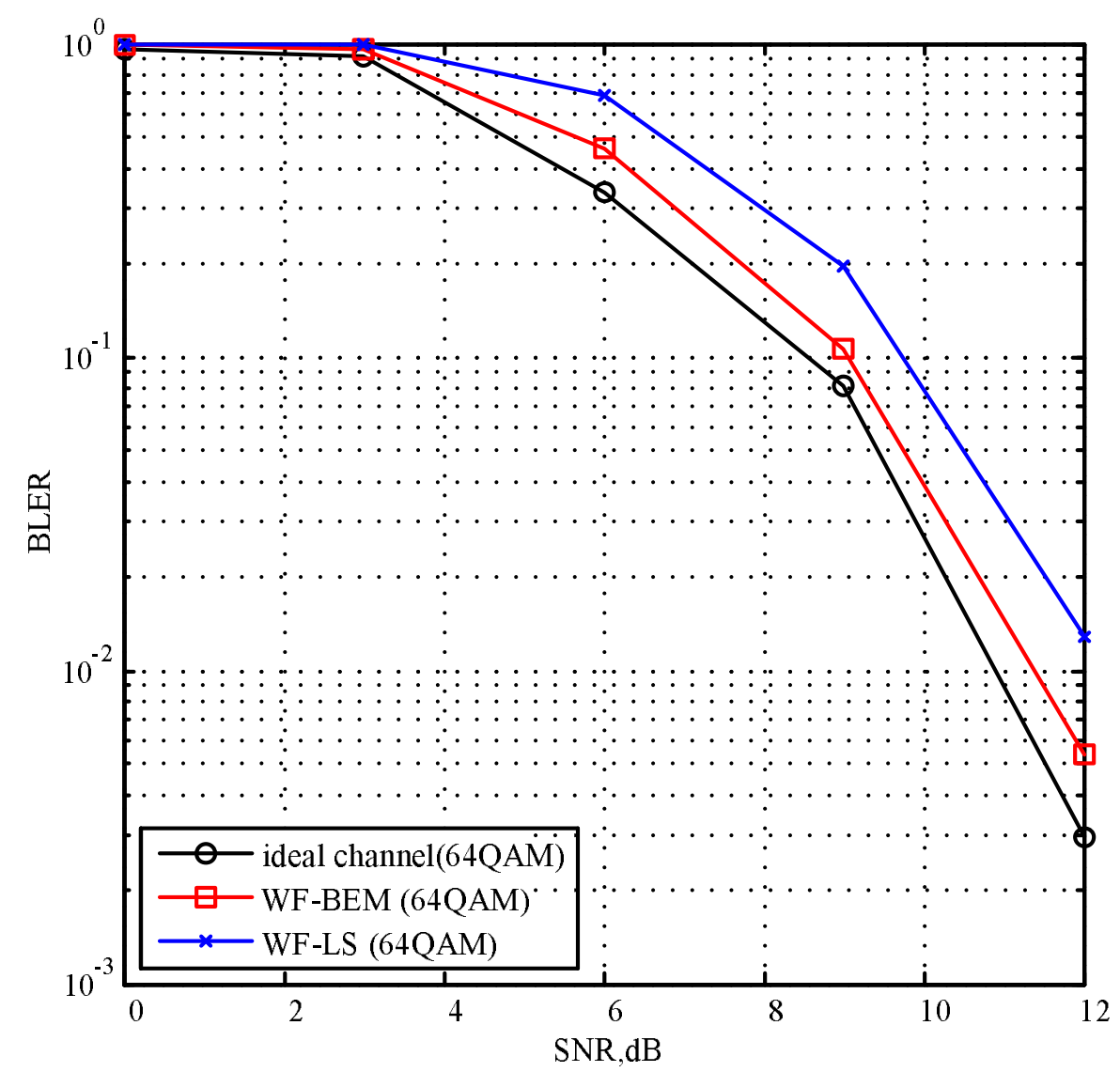

Figure 7 Performance of BLER: $30 \mathrm{~km} / \mathrm{h}, 64 Q A M$. The blue line represents the BLER of WF-LS and the red line represents that of WF-BEM. The idea case which assumes perfect channel state information is represented by the black line. 


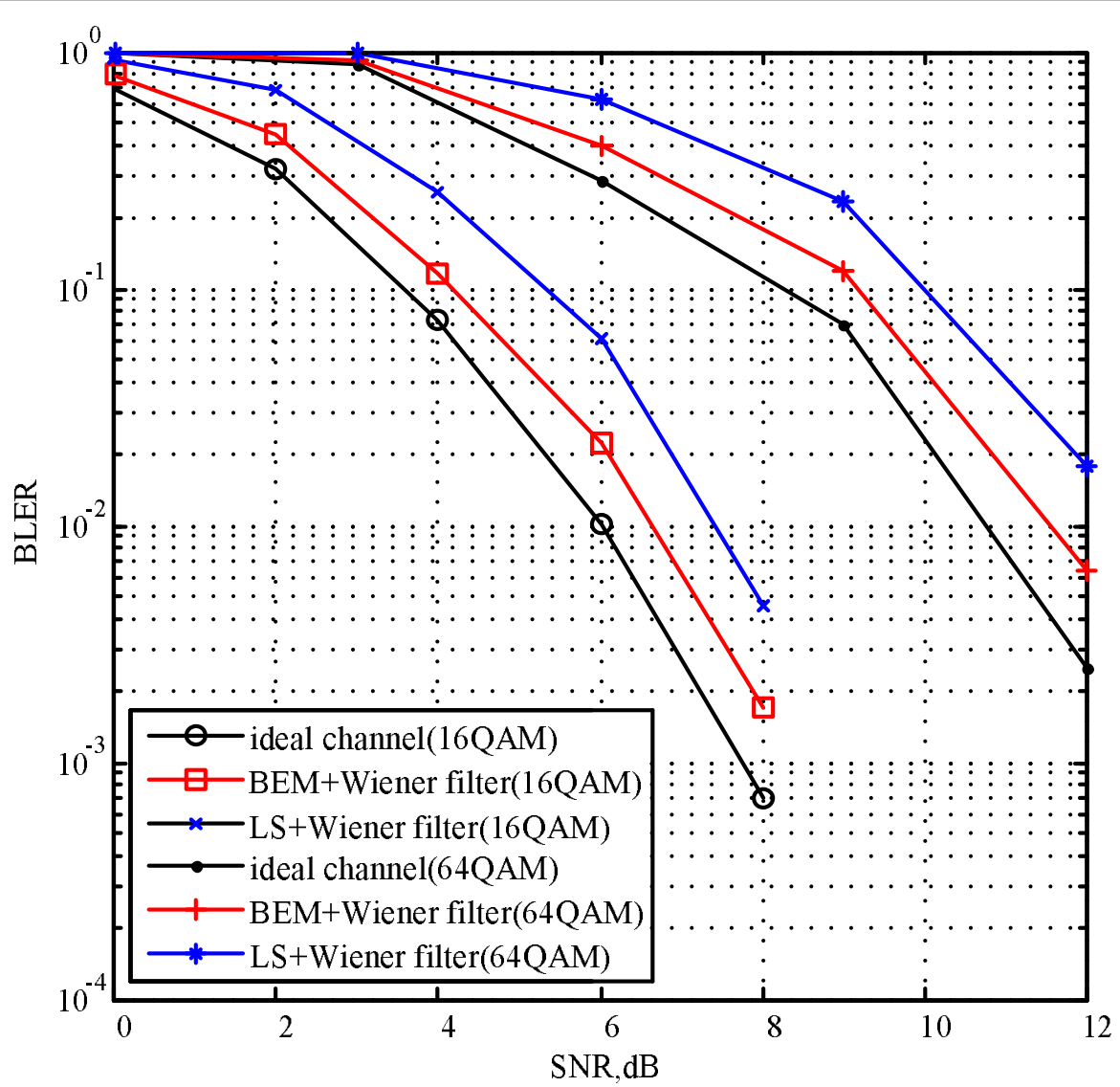

Figure 8 Performance of BLER: $120 \mathrm{~km} / \mathrm{h}, 16 Q A M$ and 64QAM. The blue lines represent the BLER of WF-LS and the red lines represent that of WF-BEM. The idea case which assumes perfect channel state information are represented by the black lines.

WF-BEM, there is less than $1 \mathrm{~dB}$ SNR gain for WF-BEM in this situation. The actual values of these estimates (i.e., ideal channel) are also given to facilitate comparison with the estimates. We can see that the proposed WF-BEM is very close to the ideal channel case, there is only less than $0.5 \mathrm{~dB}$ loss compared to the ideal one.

Figure 8 illustrates that since the wireless channel changes not that fast enough during an OFDM symbol period at $120 \mathrm{~km} / \mathrm{h}$ compared with that at 350 and 480 $\mathrm{km} / \mathrm{h}$, the performance gap between WF-BEM and WFLS is still not that obvious, there is only about $1 \mathrm{~dB}$ SNR gain for WF-BEM compared with WF-LS in this situation (for both 16 and 64QAM). And still, there is only less than $1 \mathrm{~dB}$ gap between the proposed WF-BEM and the ideal channel case.

However, in high-speed environments (350 and 480 $\mathrm{km} / \mathrm{h}$ ), the wireless channel changes quickly and drastically. Hence, in these cases the wireless channel can not be regarded as nearly static and even slow changing during one OFDM symbol period anymore. Therefore, at mobile speed of $350 \mathrm{~km} / \mathrm{h}$, compared with WF-LS, about 2.5 and $3.5 \mathrm{~dB}$ SNR gain can be obtained in 16 and 64QAM modulation mode respectively through WF-BEM, as can be seen from Figure 9. It is observed from Figure 9 that even at $350 \mathrm{~km} / \mathrm{h}$, compared to the ideal channel case, there is only about $1.5 \mathrm{~dB}$ loss of the proposed WF-BEM in 16QAM modulation mode and about less than $3 \mathrm{~dB}$ loss in 64QAM mode. From Figure 9 we can infer that the use of efficient multilevel modulations, which make use of multiple signal phase and amplitude levels to carry multiple bits per symbol, may demand more precise channel estimation to demodulate the received signal, especially in high-speed environments.

Finally, the performance of WF-BEM with WF-LS at extremely high speed $480 \mathrm{~km} / \mathrm{h}$ is presented in Figure 10. As can be observed from Figure 10, the performance of both WF-BEM and WF-LS rapidly degrade for an increasing speed. However, our proposed WF-BEM is still much better than WF-LS in 16QAM modulation mode, there is more than $3 \mathrm{~dB}$ SNR gain. There are two reasons for this degradation. Firstly, the wireless channel is changing so fast that by merely interpolating to obtain the channel estimate of data symbols becomes difficult 


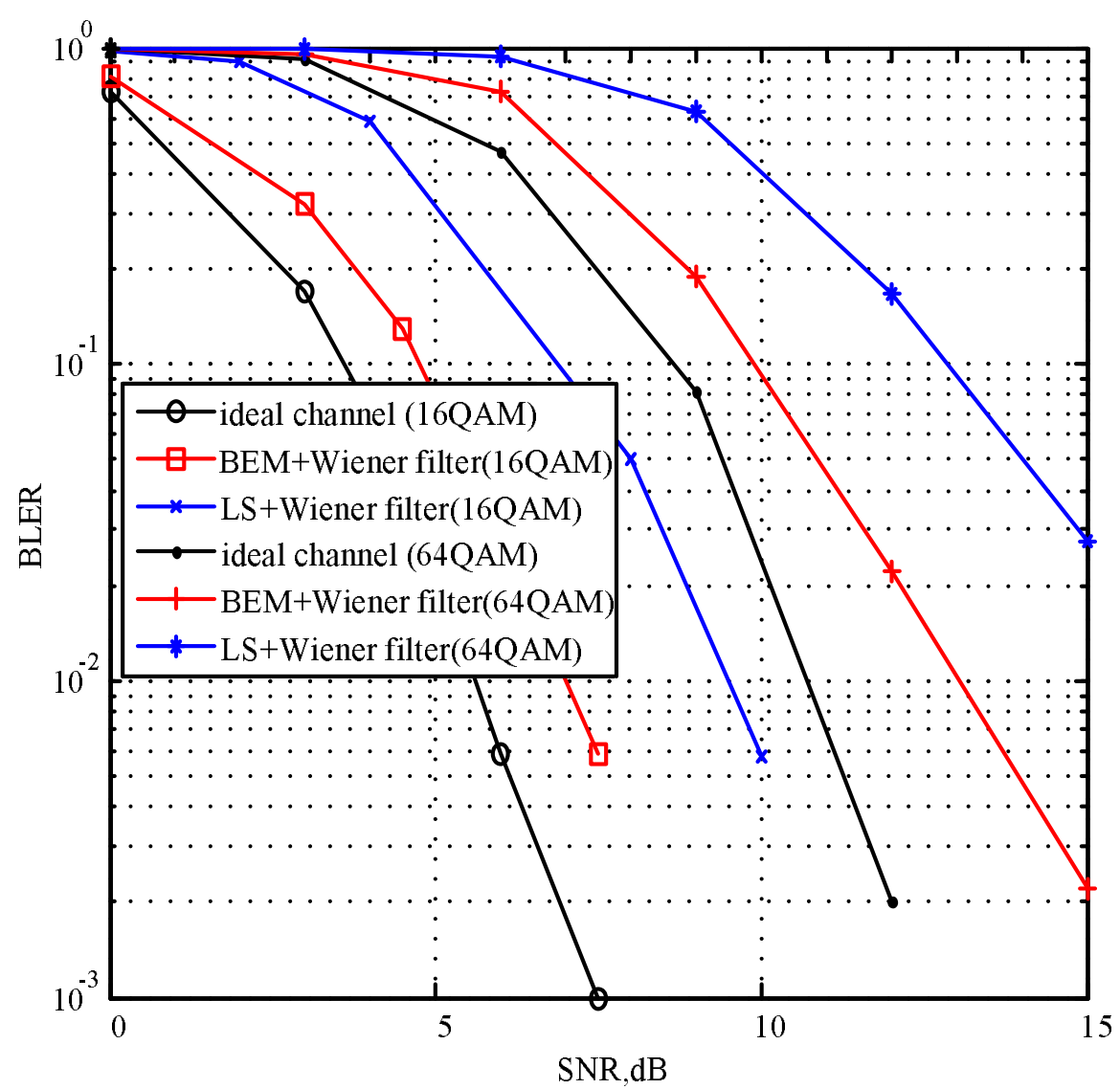

Figure 9 Performance of BLER: $350 \mathrm{~km} / \mathrm{h}$, 16QAM and 64QAM. The blue lines represent the BLER of WF-LS and the red lines represent that of WF-BEM. The idea case which assumes perfect channel state information are represented by the black lines.

and inaccurate. In this case we may need to insert pilot tones in data symbols (e.g., comb-type pilot pattern) to actually estimate the channel at all OFDM symbols. Secondly, the ICI is so evident that in this case it can not be just "equalized out" as explained above. In this case, we may have to turn to ICI-cancelation schemes (e.g., self ICI cancelation [25], the use of windowing [26] or the use of pilot tones $[27,28])$ to mitigate or suppress the ICI. Nevertheless, to change the pilot pattern at the transmitter or to implement ICI-cancelation scheme at the receiver will necessitate altering the transceiver's structure, increasing the computational complexity significantly, and moreover, the use of more pilot tones will inevitably decrease the capacity and/or throughput of the overall system. Hence, considering that the sensitivity of $M$-QAM-OFDM signals to Doppler frequency increases significantly with the alphabet size $M$, we recommend that in order to strike a compromise between complexity and performance as well as data transmission rate, low level modulation such as 8PSK, QPSK or BPSK modulation schemes should be adopted at $480 \mathrm{~km} / \mathrm{h}$. When operating under (including) 350 $\mathrm{km} / \mathrm{h}$ the use of high level modulation 16 or 64QAM can be adopted to increase data transmission rate while at the same time maintain good performance.

\section{$5.3 \mathrm{ICl}$ analysis}

In Figure 11, we show the effect of ICI that is included in the derived result (see expression (26)). It is observed from Figure 11 that when the Normalized Doppler frequency is small, the effect of ICI is also small, which may indeed be ignored just as the authors of $[11,12]$ do. However, as the moving speed increases, the Normalized Doppler frequency will increase as well, and thus the effect of ICI can not be ignored anymore in this situation. In conclusion, the effect of ICI and the noise variance will contribute together to influence the performance of OFDM systems in time-varying channels, especially in high-speed cases.

\section{Conclusions}

In this article, we have further analyzed WF-LS, including the asymptotical relationship between WF-LS with orders 2 and linear interpolation, the asymptotical 


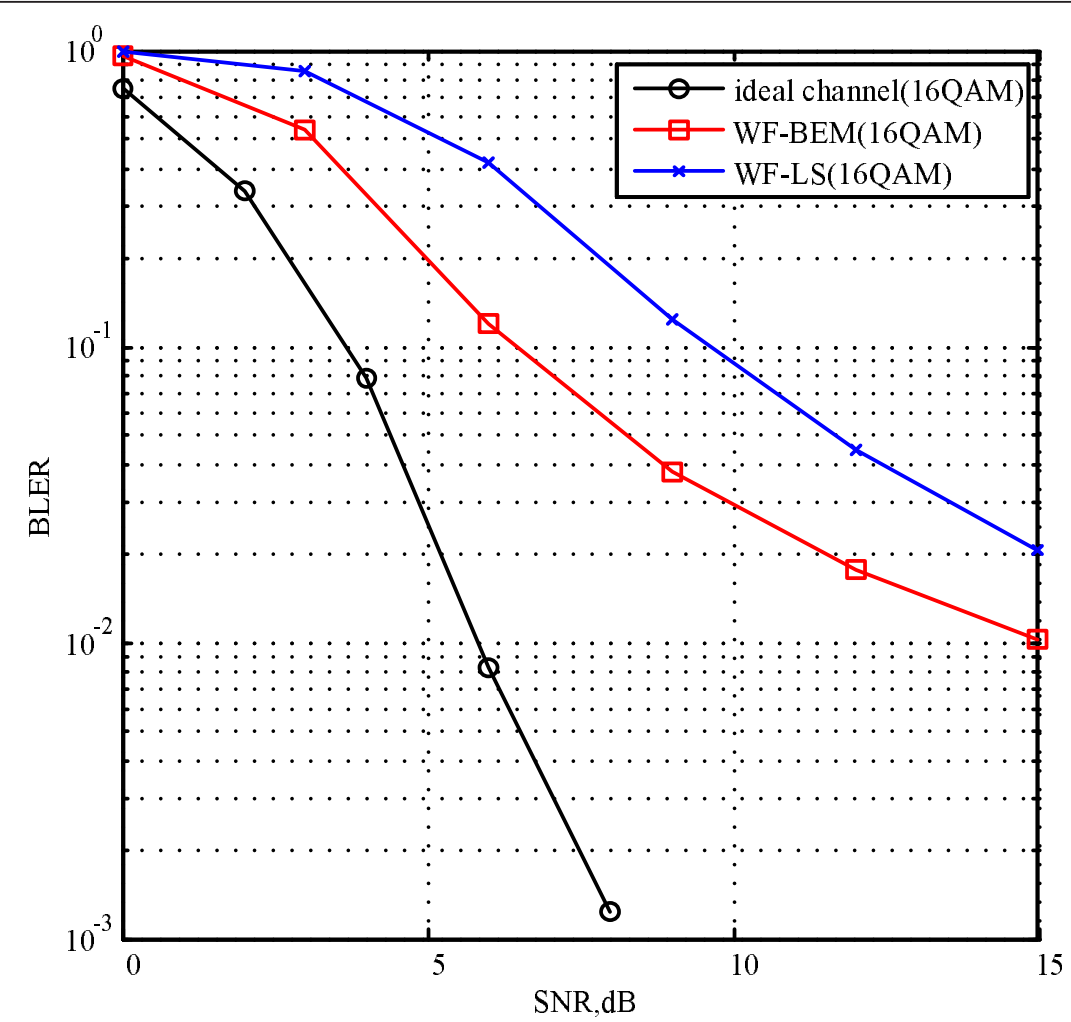

Figure 10 Performance of BLER: $480 \mathrm{~km} / \mathrm{h}, 16 Q A M$. The blue line represents the BLER of WF-LS and the red line represents that of WF-BEM. The idea case which assumes perfect channel state information is represented by the black line.

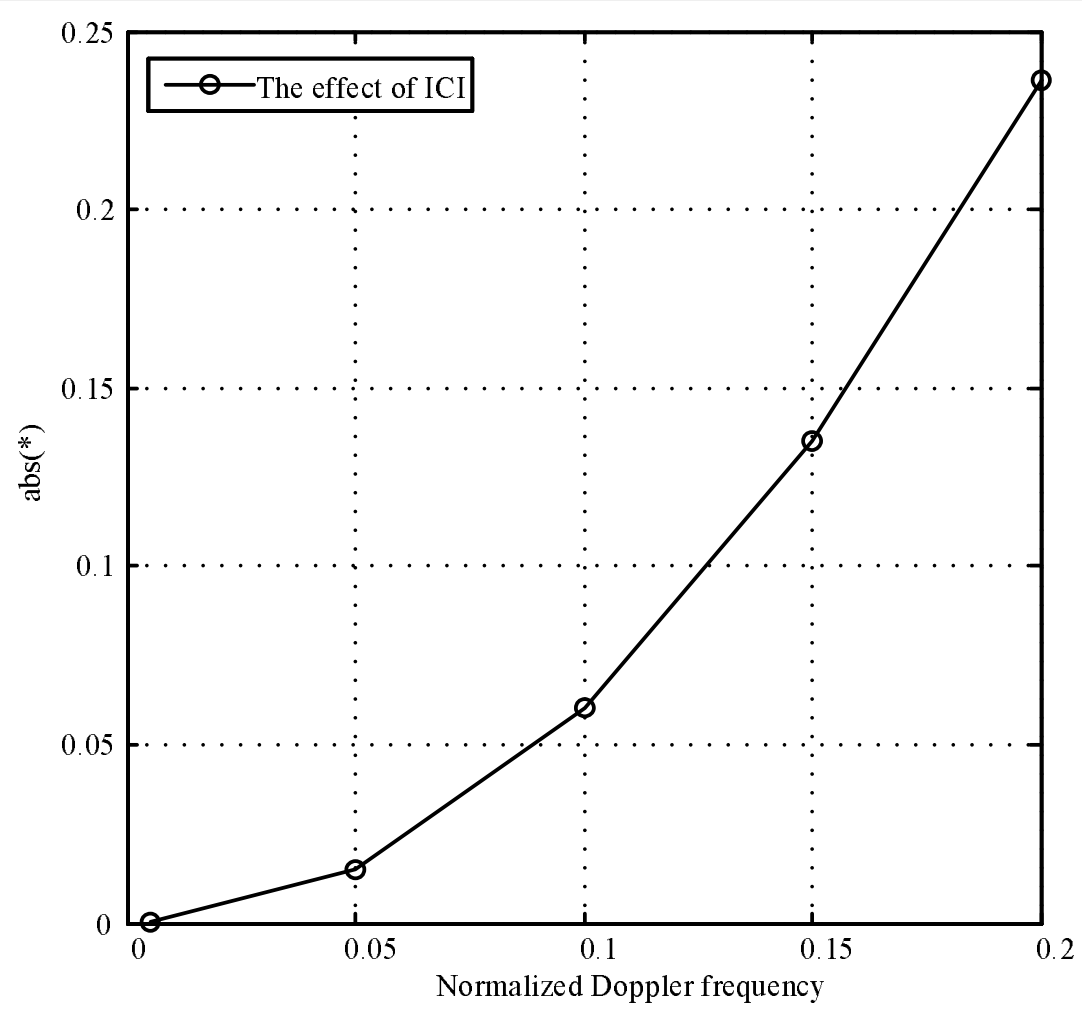

Figure 11 The effect of ICl. The effect of ICl is plotted as the absolute value versus the normalized Doppler frequency. 
relationships among WF-LS with different orders. Considering the limitation of WF-LS is that LS based channel estimation assumes the channel coefficients within one OFDM symbol period are constants, we propose to combine BEM based channel estimation, which can accurately estimate the time-varying channel coefficients during one OFDM symbol period, with WF to deal with the challenging problem of channel estimation in OFDM systems operating under various mobile environments, especially in high-speed cases. The expression for WF-BEM based channel estimation has been derived, and under the hypothesis of approximating the ICI term as additive white Gaussian noise, the derived result explicitly considers the effect of ICI. The simulation results showed that the proposed WF-BEM provides a substantial gain, in terms of estimation accuracy in time-varying channels, with respect to WF-LS, especially in fast time-varying channels.

\section{Acknowledgements}

This work was supported in part by the Chinese Important National Science \& Technology Specific Projects under Grant 2011ZX03001-007-01 and the National Science Foundation of China under Grant nos. 61032002, 60972029 and 60902026

\section{Competing interests}

The authors declare that they have no competing interests.

Received: 16 January 2012 Accepted: 31 May 2012

Published: 31 May 2012

\section{References}

1. ETSI TS 101475 V1.2.2, ETSI Broadband Radio Access Networks (BRAN), HIPERLAN Type2: Physical Layer (Aug 1999)

2. DVB, A122: framing structure, channel coding and modulation for a second generation digital terrestrial television broadcasting system (DVB-T2)

3. IEEE, Part 16: air interface for fixed and mobile broadband wireless access systems - amendment 2: physical and medium access control layers for combined fixed and mobile operation in licensed bands (2005)

4. S Sesia, I Toufik, M Baker, LTE-The UMTS Long Term Evolution-From Theory to Practice, (Wiley Press, Chichester, 2009)

5. B Muquet, M de Courville, P Duhame, Subspace-based blind and semi-blind channel estimation for OFDM systems. IEEE Trans Signal Process. 50(7), 1699-1712 (2002). doi:10.1109/TSP.2002.1011210

6. C Li, S Roy, Subspace-based blind channel estimation for OFDM by exploiting virtual carriers. IEEE Trans Wirel Commun. 2(1), 141-150 (2003). doi:10.1109/TWC.2002.806383

7. S Chen, $\mathrm{T}$ Yao, Intercarrier interference suppression and channel estimation for OFDM system in time-varying frequency-selective fading channels. IEEE Trans Consum Electron. 50(2), 429-435 (2004). doi:10.1109/TCE.2004.1309404

8. JJ van de Beek, O Edfors, M Sandell, SK Wilson, PO Börjesson, On channel estimation in OFDM systems, in Proc IEEE VTC'1995, Chicago, USA, 2 815-819 (1995)

9. YP Zhao, AP Huang, A Novel channel estimation method for OFDM mobile communication systems based on pilot signals and transform-domain processing, in Proc IEEE VTC'1997, Phoenix, AZ, 3, 2089-2093 (1997)

10. P Hoeher, S Kaiser, P Robertson, Two-dimensional pilot-symbol-aided channel estimation by Wiener filtering, in Proc IEEE Int conf on Acoustic, Speech, and Signal Processing (ICASSP), Munich, 3, 1845-1848 (1997)

11. X Dong, W Lu, ACK Soong, Linear interpolation in pilot symbol assisted channel estimation for OFDM. IEEE Trans Wirel Commun. 6(5), 1910-1920 (2007)
12. YR Zheng, CS Xiao, Channel estimation for frequency-domain equalization of single-carrier broadband wireless communications. IEEE Trans Veh Technol. 58(2), 815-82 (2009)

13. X Ma, GB Giannakis, S Ohno, Optimal training for block transmissions over doubly selective wireless fading channels. IEEE Trans Signal Process. 51(5), 1351-1366 (2003). doi:10.1109/TSP.2003.810304

14. Z Tang, RC Cannizzaro, G Leus, P Banelli, Pilot-assisted time-varying channel estimation for OFDM systems. IEEE Trans Signal Process. 55(5), 2226-2238 (2007)

15. 3GPP TS36.211 v8.7.0, Evolved Universal Terrestrial Radio Access (E-UTRA); physical channels and modulation (release 8) (May 2009)

16. X Ma, GB Giannakis, Maximum-diversity transmissions over doubly selective wireless channels. IEEE Trans Inf Theory. 49(7), 1832-1840 (2003). doi:10.1109/TIT.2003.813485

17. X Ma, MK Oh, GB Giannakis, DJ Park, Hopping pilots for estimation of frequency-offsets and multi-antenna channels in MIMO OFDM. IEEE Trans Commun. 53(1), 162-172 (2005). doi:10.1109/TCOMM.2004.840663

18. MK Ozdemir, H Arslan, Channel estimation for wireless OFDM systems. IEEE Commun Surv Tut. 9(2), 18-48 (2007)

19. A Ancora, C Bona, DTM Slock, Down-sampled impulse response leastsquares channel estimation for LTE OFDMA, in Proc IEEE Intl conf on Acoustic, Speech, and Signal Processing (ICASSP), Honolulu, HI, 3, 293-296 (2007)

20. Y Mostofi, DC Cox, ICI mitigation for pilot-aided OFDM mobile systems. IEEE Trans Wirel Commun. 4(2), 765-774 (2005)

21. H Stark, JW Woods, Probability and Random Processes with Applications to Signal Processing, (Prentice Hall, Upper Saddle River, 2002)

22. 3GPP TS36.212 v8.7.0, Evolved Universal Terrestrial Radio Access (E-UTRA); multiplexing and channel coding (release 8) (May 2009)

23. JJ van de Beek, PO Börjesson, M-L Boucheret, D Landström, J Arenas, P Ödling, C Östberg, M Wahlqvist, S Wilson, A time and frequency synchronization scheme for multiuser OFDM. IEEE J Sel Areas Commun. 17(11), 1900-1914 (1999). doi:10.1109/49.806820

24. M Morelli, Timing and frequency synchronization for the uplink of an OFDMA system. IEEE Trans Commun. 52(2), 296-306 (2004). doi:10.1109/ TCOMM.2003.822699

25. Y Zhao, S-G Häaggman, Sensitivity to Doppler shift and carrier requency errors in OFDM systems-the consequences and solutions, in Proc IEEE VTC'1996, Atlanta, GA, 3, 1564-1568 (1996)

26. C Muschallik, Improving an OFDM reception using an adaptive Nyquist windowing. IEEE Trans Consumer Electron. 42, 259-269 (1996). doi:10.1109/ 30.536046

27. SW Hou, CC Ko, Intercarrier interference suppression for OFDMA uplink in time- and frequency-selective fading channels. IEEE Trans Veh Technol. 58(6), 2741-2754 (2009)

28. X Huang, $\mathrm{H}$ Wu, Robust and efficient intercarrier interference mitigation for OFDM systems in time-varying fading channels. IEEE Trans Veh Technol. 56(5), 2517-2528 (2007)

doi:10.1186/1687-1499-2012-186

Cite this article as: Zhong et al: Channel estimation in OFDM systems operating under high mobility using Wiener filter combined basis expansion model. EURASIP Journal on Wireless Communications and Networking 2012 2012:186.

\section{Submit your manuscript to a SpringerOpen ${ }^{\circ}$ journal and benefit from:}

- Convenient online submission

- Rigorous peer review

- Immediate publication on acceptance

- Open access: articles freely available online

- High visibility within the field

- Retaining the copyright to your article

Submit your next manuscript at $>$ springeropen.com 